\title{
BMJ Open Optimising gestational weight gain and improving maternal and infant health outcomes through antenatal dietary, lifestyle and physical activity advice: the OPTIMISE randomised controlled trial protocol
}

Jodie M Dodd, ${ }^{1,2}$ Andrea R Deussen, ${ }^{1}$ Jennie Louise ${ }^{3}$

To cite: Dodd JM, Deussen AR, Louise J. Optimising gestational weight gain and improving maternal and infant health outcomes through antenatal dietary, lifestyle and physical activity advice: the OPTIMISE randomised controlled trial protocol. BMJ Open 2018;8:e019583. doi:10.1136/ bmjopen-2017-019583

- Prepublication history for this paper is available online To view these files, please visit the journal online (http://dx.doi org/10.1136/bmjopen-2017019583).

Received 12 September 2017 Revised 10 January 2018 Accepted 11 January 2018

Check for updates

${ }^{1}$ Discipline of Obstetrics and Gynaecology, Robinson Research Institute, The University of Adelaide, Adelaide, South Australia, Australia

${ }^{2}$ Department of Perinatal Medicine, Women's and Children's Hospital, North Adelaide, South Australia, Australia

${ }^{3}$ School of Public Health, The University of Adelaide, Adelaide, South Australia, Australia

Correspondence to

Professor Jodie M Dodd; jodie.dodd@adelaide.edu.au

\section{ABSTRACT}

Introduction Obesity represents a significant health burden, and WHO recognises the importance of preventing weight gain and subsequent development of obesity among adults who are within the healthy weight range. Women of reproductive age have demonstrated high rates of weight gain during pregnancy placing them at risk of becoming overweight or obese. We will evaluate the effects of dietary and physical activity advice on maternal, fetal and infant health outcomes, among pregnant women of normal body mass index (BMI).

Methods and analysis We will conduct a randomised controlled trial, consenting and randomising women with a live singleton pregnancy between $10^{+0}$ and $20^{+0}$ weeks and BMI $18.5-24.9 \mathrm{~kg} / \mathrm{m}^{2}$ at first antenatal visit, from a tertiary maternity hospital. Women randomised to the Lifestyle Advice Group will receive three face-to-face sessions (two with a research dietitian and one with a trained research assistant) and three telephone calls over pregnancy, in which they will be provided with dietary and lifestyle advice and encouraged to make change using a SMART goals approach. Women randomised to the Standard Care Group will receive routine antenatal care. The primary outcome is infant birth weight $>4 \mathrm{~kg}$. Secondary outcomes will include adverse infant and maternal outcomes, maternal weight change, maternal diet and physical activity changes, maternal quality of life and emotional well-being, fetal growth and costs of healthcare. We will recruit 624 women to detect a reduction from $8.72 \%$ to $3.87 \%$ (alpha 0.05 (two-tailed); power 70\%) in infants with birth weight $>4 \mathrm{~kg}$. Analyses will be intention to treat with estimates reported as relative risks and $95 \% \mathrm{Cls}$.

Ethics and dissemination Ethical approval has been obtained from the Women's and Children's Hospital ethics committee. Findings will be disseminated widely via journal publication and conference presentation(s), and participants informed of results.

Trial registration number ACTRN12614000583640.

\section{INTRODUCTION}

Worldwide, more than 1.9 billion adults and 41 million children under the age of 5 years
Strengths and limitations of this study

- Robust evaluation of the effects of a dietary and lifestyle intervention during pregnancy for women of normal body mass index (BMI).

- Prospectively measured and calculated height, weight and BMI at the time of the first antenatal booking visit.

- A wide range of clinically relevant maternal and infant health outcomes will be reported.

are overweight or obese. ${ }^{1}$ Obesity represents a significant healthcare burden, with WHO recognising the importance of preventing weight gain among adults who are within the healthy weight range. ${ }^{2}$ Women of reproductive age have demonstrated high rates of gestational weight gain (GWG) during pregnancy and postpartum weight retention (PPWR), ${ }^{3}$ and therefore represent an 'at-risk' population.

The Institute of Medicine (IoM) has reviewed the considerable observational literature which is available, recommending GWG between 11.5 and $16.0 \mathrm{~kg}$ for pregnant women of normal body mass index (BMI), defined as BMI between 18.5 and $24.9 \mathrm{~kg} /$ $\mathrm{m}^{2}$. Weight gain in excess of these recommendations is well recognised as a significant risk factor for adverse maternal and infant outcomes during pregnancy and childbirth, even among women of normal BMI, ${ }^{5-8}$ and more than doubles a woman's risk of PPWR. ${ }^{9}$ Indeed, more than $70 \%$ of women with a BMI retain more than $5 \mathrm{~kg}$ weight up to 1 year after birth, ${ }^{10} 11$ contributing to an increase in interpregnancy BMI, an increased risk of adverse outcomes in a subsequent pregnancy and longer-term risk of obesity. ${ }^{12-14}$ There is 
also increasing evidence that excessive GWG increases the chance of high infant birth weight, with both factors independently associated with an increased risk of child obesity ${ }^{15-17}$ Specifically, the risk of early childhood obesity increases by a factor of 1.08 (95\% CI 1.03 to 1.14 ) per kilogram of maternal weight gained above the recommendations. ${ }^{18}$ All of these factors combine to create a vicious cycle in which the intergenerational effects of obesity are perpetuated. $^{19}$

While there is extensive observational literature defining the pregnancy and childbirth complications associated with excessive GWG and PPWR for women of normal BMI, there is limited information describing effective interventions. Our systematic review of the literature identified 12 randomised controlled trials (RCTs) of 2713 pregnant women, who were recruited across all BMI categories. ${ }^{20}$ Of these trials, eight specifically identified 1048 women to be of normal BMI, of which only five reported clinical outcomes for women of normal BMI (714 women).${ }^{20}$ Women of normal BMI who received an antenatal intervention gained less weight $(1.25 \mathrm{~kg} ; 95 \% \mathrm{CI}$ -2.39 to -0.11$)$ than those women receiving standard antenatal care and were less likely to have weight gain above the IoM recommendations (relative risk (RR) 0.72; $95 \%$ CI 0.60 to 0.86 ; five studies, 714 women).$^{20}$ However, the effect on clinical pregnancy outcomes was less clear (two studies, 243 women), and only two studies reported PPWR at 8 weeks and 6 months after birth. ${ }^{20}$ While there was a modest overall effect on GWG, effects on clinical pregnancy and birth outcomes have been poorly reported to date, and there is no information relating to longerterm effects on both maternal and child health.

The aims of the OPTIMISE randomised trial are to evaluate the effects of dietary and physical activity advice on maternal, fetal and infant health outcomes, among pregnant women of normal BMI.

\section{METHODS AND ANALYSIS \\ Study design \\ RCT.}

\section{Inclusion criteria}

Women with a singleton, live gestation between $10^{+0}$ and $20^{+0}$ weeks who are of normal BMI (BMI 18.5 to $24.9 \mathrm{~kg} / \mathrm{m}^{2}$ ) at their first antenatal visit will be eligible to participate.

\section{Exclusion criteria}

Women with a multiple pregnancy or who have type 1 or 2 diabetes who were diagnosed prior to pregnancy will be excluded from participation.

\section{Trial entry}

Eligible women will be identified in the antenatal clinic of the Women's and Children's Hospital, given the trial information sheet and counselled by a researcher, before obtaining informed written consent. Randomisation will occur using the computer-based randomisation service in the Discipline of Obstetrics and Gynaecology, The University of Adelaide. Recruitment commenced in June 2014, and the study is ongoing.

The randomisation schedule will use balanced variable blocks and will be prepared by an investigator not involved with recruitment or clinical care. There will be stratification of women according to parity ( 0 vs 1 or more). Eligible women will be randomised to either the Lifestyle Advice Group or the Standard Care Group. Blinding of participants is not possible given the nature of the intervention. Where possible, antenatal care providers, outcome assessors and data analysts will be blinded to treatment allocation.

\section{Treatment schedules}

Women who are randomised to the Lifestyle Advice Group will receive three individual face-to-face sessions during pregnancy, with two provided by the dietitian shortly after trial entry and again at 28 weeks of gestation, and one provided by a research assistant at 36 weeks of gestation. Women will also receive three telephone calls from the research assistant at 20, 24 and 32 weeks of gestation. The dietary advice provided will be individually tailored, consistent with current Australian dietary standards, ${ }^{21}$ and based on our experience with the LIMIT randomised trial. ${ }^{22}{ }^{23}$ Recommendations and dietary targets will be individualised, taking into account each woman's basal metabolic rate and activity levels. ${ }^{24}$ The dietary intervention will maintain a balance of carbohydrates, fat and protein, while specifically encouraging women to reduce their intake of energy dense and non-core foods high in refined carbohydrates and saturated fats, while increasing their intake of fibre, and to consume two serves of fruit, five serves of vegetables and three serves of dairy each day. ${ }^{21-23}$

Tailoring of the intervention will be informed by stage theories of health decision making that propose that individuals progress through a series of cognitive phases when undertaking behavioural change. ${ }^{25}$ Initially, there will be a planning session with a research dietitian, in which women will be provided with written dietary and activity information, an individual diet and physical activity plan, recipe book and example menu plans. Women will be encouraged to set achievable goals for dietary and physical activity change, supported to make these lifestyle changes and to self-monitor their progress, using a SMART goals approach. Women will be encouraged to involve their partner or significant support person in these sessions. These principles will be reinforced at subsequent faceto-face visits with the dietitian and research assistant and during the telephone contacts. ${ }^{22} 23$

Women who are randomised to the Standard Care Group will receive their antenatal care according to their local hospital practice, which does not currently include information relating to dietary intake and physical activity during pregnancy. 


\section{Ongoing follow-up of all women in both treatment groups}

All women will be asked to complete a food frequency questionnaire, physical activity diary and quality of life assessments at trial entry, 28 and 36 weeks of gestation and 6 months postpartum. Their weight will be recorded at trial entry, at 28 weeks of gestation and at 36 weeks of gestation or nearest to birth. All women will be encouraged to attend for a research ultrasound at 28 and 36 weeks of gestation that does not constitute routine clinical care to monitor fetal growth and well-being.

After birth, information will be obtained relating to birth and infant outcomes from the case notes by the research assistant, who will remain blinded to the woman's allocated treatment group.

\section{Study endpoints}

The primary trial outcome is the incidence of infants with birth weight $>4 \mathrm{~kg}$.

The secondary study outcomes are

1. adverse outcomes for the infant including preterm birth (birth before 37 weeks of gestation), mortality (either stillbirth (intrauterine fetal death after trial entry and prior to birth) or infant death (death of a live born infant prior to hospital discharge and excluding lethal congenital anomalies)), infant birth weight, infant birth weight $<2500 \mathrm{~g}$, infant birth weight $>4500 \mathrm{~g}$, large for gestational age defined as infant birth weight $>90$ th percentile for gestational age and infant sex, small for gestational age defined as infant birth weight $<10$ th percentile for gestational age and infant sex, hypoglycaemia requiring intravenous treatment, admission to neonatal intensive care unit, or special care baby unit, hyperbilirubinaemia requiring phototherapy, nerve palsy, fracture, birth trauma, shoulder dystocia, corticosteroid use, respiratory distress syndrome (with moderate or severe respiratory disease defined as mean airway pressure $>10 \mathrm{~cm} \mathrm{H}_{2} \mathrm{O}$ and/or fractional inspired oxygen $>0.80$ with ventilation), ${ }^{26}$ discharge home on oxygen, patent ductus arteriosus, proven systemic infection requiring treatment, retinopathy of prematurity, necrotising enterocolitis, neonatal encephalopathy, ${ }^{27}$ seizures, length of stay and infant exclusively breast fed at hospital discharge;

2. adverse outcomes for the woman including maternal hypertension and pre-eclampsia (in accordance with recognised Australasian Society for the Study of Hypertension in Pregnancy criteria), ${ }^{28}$ maternal gestational diabetes, need for and length of antenatal hospital stay, antepartum haemorrhage requiring hospitalisation, preterm prelabour ruptured membranes, chorioamnionitis, need and reason for induction of labour, any antibiotic use during labour, caesarean section, postpartum haemorrhage (blood loss $\geq 600 \mathrm{~mL}$ ), perineal trauma, wound infection, endometritis, length of postnatal hospital stay, thromboembolic disease and maternal death;
3. maternal weight changes including total GWG, average weekly gestational gain, GWG below/within/ above IoM recommendations, ${ }^{4}$ maternal weight at 6 , 12 and 18-24 months after birth to calculate PPWR, return to early pregnancy weight and anthropometric assessment (skin-fold thickness, body circumferences and bioimpedance to assess adiposity);

4. maternal changes in diet and physical activity as measured by questionnaires completed by the woman at trial entry, 28 and 36 weeks of gestation and 6, 12 and 18-24 months after birth (Harvard Semi-quantitative Food Frequency Questionnaire ${ }^{29} 30$ and the Short Questionnaire to Assess Health-enhancing physical activity) ${ }^{31}$;

5. maternal quality of life and emotional well-being as measured by questionnaires completed by the woman at 6 months postpartum relating to quality of life (as measured using the Short Form-12 Health Survey Questionnaire), ${ }^{32}$ preferences for treatment, satisfaction with care, anxiety (as measured by the Short Form Spielberger State Trait Inventory ${ }^{33}$ ) and depression (as measured by the Edinburgh Postnatal Depression Scale ${ }^{34}$ ). Women will be asked a series of questions about their satisfaction with the intervention, using items modified from a previous childbirth questionnaire $^{35}$;

6. fetal growth and well-being at 28 and 36 weeks of gestation assessed by ultrasound (fetal biometry, estimated weight, liquor volume, umbilical artery Doppler waveform and adiposity ${ }^{36}$;

7. costs of healthcare: the primary measure of outcome for the economic analysis will be the cost per live birth. Resource use will include the provision of the dietary intervention and direct costs of healthcare (expected average clinic fees, the frequency and duration of general practitioner and antenatal visits, as well as inpatient admissions), determined by hospital outpatient visits, inpatient admissions and published data sets including Pharmaceutical Benefits Scheme (PBS), Medical Benefits Schedule (MBS) and Australian Refined Diagnosis Related Groups cost weights. Mean costs and effectiveness between treatment groups will be compared and incremental cost-effectiveness ratios and CIs presented. For varying threshold values of cost-effectiveness, acceptability curves will be presented as described previously. ${ }^{37}$ An assessment of the sensitivity of the results to variation in measured resource use, effectiveness and/or unit costs will be undertaken using appropriate oneway and multi-way sensitivity analyses, as described previously. ${ }^{37}$

\section{Sample size estimation}

The primary clinical endpoint is the incidence of infants born with birth weight $>4 \mathrm{~kg}$, with an estimated incidence in women eligible for this trial of $8.72 \% .^{38}$ To detect a difference from $8.72 \%$ to $3.89 \%$ (alpha 0.05 ; power $70 \%$ ), we will recruit a total of 624 women. This sample size will 
Table 1 Estimated differences in secondary outcomes

\begin{tabular}{lcc}
\hline Outcome & $\begin{array}{l}\text { Assumed control } \\
\text { group \% }\end{array}$ & Intervention \% \\
\hline GDM & 3.4 & 0.6 \\
$\begin{array}{l}\text { Hypertension/pre- } \\
\text { eclampsia }\end{array}$ & 4.6 & 1.3 \\
Induction of labour & 26.8 & \\
Caesarean section & 24.6 & 18.5 \\
Birth weight $<2.5 \mathrm{~kg}$ & 6.2 & 16.6 \\
\hline
\end{tabular}

GDM, gestational diabetes mellitus.

be powered $(70 \%)$ to detect the differences in secondary outcomes as detailed in table 1 below.

\section{Analysis and reporting of results}

The initial analysis will examine baseline characteristics of all randomised women, as an indication of comparable treatment groups, and include maternal age, parity, race, height, weight, smoking history, obstetric history and previous gestational diabetes. Primary and secondary outcomes will be analysed on an 'intention to treat' basis, according to treatment allocation at randomisation. RRs and $95 \%$ CIs will be reported for the major outcomes, and the number needed to treat to benefit or harm calculated. Regression techniques will examine the influence of prognostic factors on the major outcomes.

As the lead investigator, JMD will have access to the data and acts as a guarantor to the final data set. The trial manager (ARD) and statistician (JL) will have access to the data, and the statistician (JL) will be responsible for the development of the statistical analysis plan, prior to the conduct of any statistical analyses.

\section{ETHICS AND DISSEMINATION}

\section{Data management}

All information obtained from this study will remain strictly confidential. While the results of the study will be published, no data will be presented to allow the identification of individual women. Data will be stored in a locked filing cabinet or on password protected computer file and accessible only by the study team.

We have previous experience recruiting women for lifestyle intervention trials in this setting ${ }^{23}$ and will consider adding further sites as required. Women who withdraw from ongoing participation after randomisation will be asked for consent to use the data already collected prior to withdrawal, including birth outcomes where possible.

Following completion of the relevant statistical analyses, manuscripts will be prepared for publication, with subsequent presentation of results at relevant scientific and clinical meetings. Participants will be notified of the findings of the trial. The findings of this trial will contribute to the currently available literature regarding the effect of antenatal dietary and lifestyle interventions for women of normal BMI and to the development of evidence-based clinical practice guidelines.

Recruitment commenced in June 2014. We expect to have completed data collection and data cleaning by April 2018. The final results of the trial will be published in international peer-reviewed journals.

\section{Ethical approval}

A Data and Safety Monitoring Board (DSMB) will monitor efficacy (or futility) and adverse effects. Based on these considerations, the DSMB may recommend that the protocol be modified or that the OPTIMISE trial be terminated. The DSMB will consist of experts in relevant obstetrics, neonatology and research methodology. All adverse events involving women and infants enrolled in the trial will be reviewed by a multidisciplinary committee, who will be blinded to treatment allocation, in order to clarify cause. These data will be made available for the DSMB.

Contributors JMD drafted the initial protocol and manuscript. JMD, JL and ARD contributed to revisions of the protocol and manuscript and gave approval for the final version to be published. JL conducted the sample size calculation and statistical analyses and will be responsible for the completion of the final statistical analysis plan.

Funding The OPTIMISE Trial is funded by the Lloyd Cox Strategic Research Excellence Award (University of Adelaide Discipline of Obstetrics and Gynaecology and Robinson Research Institute). JMD is supported through an NHMRC Practitioner Fellowship (ID 627005).

Competing interests None declared.

Patient consent Obtained.

Ethics approval Women's and Children's Hospital Human Research ethics committee.

Provenance and peer review Not commissioned; externally peer reviewed.

Open Access This is an Open Access article distributed in accordance with the Creative Commons Attribution Non Commercial (CC BY-NC 4.0) license, which permits others to distribute, remix, adapt, build upon this work non-commercially, and license their derivative works on different terms, provided the original work is properly cited and the use is non-commercial. See: http://creativecommons.org/ licenses/by-nc/4.0/

(c) Article author(s) (or their employer(s) unless otherwise stated in the text of the article) 2018. All rights reserved. No commercial use is permitted unless otherwise expressly granted.

\section{REFERENCES}

1. World Health Organisation. Overweight and obesity fact sheet June 2016. Geneva, Switzerland: World Health Organisation, 2016.

2. World Health Organisation. Global action plan for the prevention and control of noncommunicable diseases 201-2020. Geneva, Switzerland: World Health Organisation, 2013.

3. Cameron AJ, Welborn TA, Zimmet PZ, et al. Overweight and obesity in Australia: the 1999-2000 Australian Diabetes, Obesity and Lifestyle Study (AusDiab). Med J Aust 2003;178:427-32.

4. Institute of Medicine. Weight gain during pregnancy: reexamining the guidelines. In: Rasmussen KM, Yaktine AL, eds. Washington D.C: The National Academies Press, 2009.

5. Dodd JM, Grivell RM, Nguyen AM, et al. Maternal and perinatal health outcomes by body mass index category. Aust N Z J Obstet Gynaecol 2011;51:136-40.

6. Callaway LK, Prins JB, Chang AM, et al. The prevalence and impact of overweight and obesity in an Australian obstetric population. Med J Aust 2006;184:56-9.

7. Cedergren M. Effects of gestational weight gain and body mass index on obstetric outcome in Sweden. Int J Gynaecol Obstet 2006;93:269-74. 
8. Cedergren MI. Optimal gestational weight gain for body mass index categories. Obstet Gynecol 2007;110:759-64.

9. Rong K, Yu K, Han X, et al. Pre-pregnancy BMI, gestational weight gain and postpartum weight retention: a meta-analysis of observational studies. Public Health Nutr 2015;18:2172-82.

10. Ma D, Szeto IM, Yu K, et al. Association between gestational weight gain according to prepregnancy body mass index and short postpartum weight retention in postpartum women. Clin Nutr 2015;34.

11. Nehring I, Schmoll S, Beyerlein A, et al. Gestational weight gain and long-term postpartum weight retention: a meta-analysis. Am J Clin Nutr 2011;94:1225-31.

12. Amorim AR, Rössner S, Neovius M, et al. Does excess pregnancy weight gain constitute a major risk for increasing long-term BMI? Obesity 2007;15:1278-86.

13. Kac G, Benício MH, Velásquez-Meléndez G, et al. Gestational weight gain and prepregnancy weight influence postpartum weight retention in a cohort of brazilian women. J Nutr 2004;134:661-6.

14. Parker JD, Abrams B. Differences in postpartum weight retention between black and white mothers. Obstet Gynecol 1993;81:768-74.

15. Rooney BL, Mathiason MA, Schauberger CW. Predictors of obesity in childhood, adolescence, and adulthood in a birth cohort. Matern Child Health J 2011;15:1166-75.

16. Oken E, Taveras EM, Kleinman KP, et al. Gestational weight gain and child adiposity at age 3 years. Am J Obstet Gynecol 2007;196:322-8.

17. Wrotniak BH, Shults J, Butts S, et al. Gestational weight gain and risk of overweight in the offspring at age $7 \mathrm{y}$ in a multicenter, multiethnic cohort study. Am J Clin Nutr 2008;87:1818-24.

18. Schack-Nielsen L, Michaelsen KF, Gamborg M, et al. Gestational weight gain in relation to offspring body mass index and obesity from infancy through adulthood. Int J Obes 2010;34:67-74.

19. Cnattingius S, Villamor E, Lagerros YT, et al. High birth weight and obesity--a vicious circle across generations. Int $\mathrm{J}$ Obes 2012;36:1320-4.

20. O'Brien CM, Grivell RM, Dodd JM. Systematic review of antenatal dietary and lifestyle interventions in women with a normal body mass index. Acta Obstet Gynecol Scand 2016;95:259-69.

21. The Department of Health. The Australian guide to healthy eating. http://www.health.gov.au/internet/main/publishing.nsf/content/ health-pubhlth-publicat-document-fdcons-cnt.htm

22. Dodd JM, Cramp C, Sui Z, et al. The effects of antenatal dietary and lifestyle advice for women who are overweight or obese on maternal diet and physical activity: the LIMIT randomised trial. BMC Med 2014;12:161.

23. Dodd JM, Turnbull D, McPhee AJ, et al. Antenatal lifestyle advice for women who are overweight or obese: LIMIT randomised trial. BMJ 2014;348:g1285.
24. Logue J, Thompson L, Romanes F, et al. Management of obesity: summary of SIGN guideline. BMJ 2010;340:c154.

25. Bennett P, Murphy S. Psychology and health promotion. Buckingham: Open University Press, 1997.

26. Australian and New Zealand Neonatal Nurses. ANZNN data dictionary 2009. 2009. http://www.preru.unsw.edu.au/PRERUWeb. nsf/page/ANZNN

27. Sarnat HB. Neonatal encephalopathy following fetal distress. Arch Neurol 1976;33:696-705.

28. Brown MA, Hague WM, Higgins J, et al. The detection, investigation and management of hypertension in pregnancy: full consensus statement. Aust N Z J Obstet Gynaecol 2000;40:139-55

29. Willett WC, Reynolds RD, Cottrell-Hoehner S, et al. Validation of a semi-quantitative food frequency questionnaire: comparison with a 1-year diet record. J Am Diet Assoc 1987;87:43-7.

30. Ibiebele TI, Parekh S, Mallitt KA, et al. Reproducibility of food and nutrient intake estimates using a semi-quantitative FFQ in Australian adults. Public Health Nutr 2009;12:2359-65.

31. Wendel-Vos GC, Schuit AJ, Saris WH, et al. Reproducibility and relative validity of the short questionnaire to assess health-enhancing physical activity. J Clin Epidemiol 2003;56:1163-9.

32. Ware JE, Sherbourne CD. The MOS 36-item short-form health survey (SF-36). I. Conceptual framework and item selection. Med Care 1992;30:473-83.

33. Marteau TM, Bekker $\mathrm{H}$. The development of a six-item short-form of the state scale of the Spielberger State-Trait Anxiety Inventory (STAI). Br J Clin Psychol 1992;31:301-6.

34. Cox JL, Holden JM, Sagovsky R. Detection of postnatal depression - development of the 10 item Edinburgh Postnatal Depression Scale (EDPS). Brit J Psych 1987;154:782-6.

35. Dodd JM, Newman A, Yelland LN, et al. Effects of antenatal lifestyle advice for women who are overweight or obese on maternal quality of life: the LIMIT randomised trial. Acta Obstet Gynecol Scand 2016;95:259-69.

36. Grivell RM, Yelland LN, Deussen A, et al. Antenatal dietary and lifestyle advice for women who are overweight or obese and the effect on fetal growth and adiposity: the LIMIT randomised trial. BJOG 2016;123:233-43.

37. Dodd JM, Ahmed S, Karnon J, et al. The cost-effectiveness of providing antenatal lifestyle advice for women who are overweight or obese: the LIMIT randomised trial. BMC Obes 2015;2:14.

38. Scheil W, Scott J, Catcheside B, et al. Pregnancy outcome in South Australia 2014. Pregnancy Outcome Unit SA Health, Adelaide: Government of South Australia, 2016. 